\title{
Research of rice crops in Krasnodar region by remote sensing data
}

\author{
Sergey Garkusha ${ }^{1}$, Mikhail Skazhennik ${ }^{1, *}$, Evgeny Kiselev $^{2}$, Vitaliy Chizhikov ${ }^{1}$, and Alexey \\ Petrushin $^{3}$ \\ ${ }^{1}$ Federal Scientific Rice Centre, 3, Belozerny, 350921, Krasnodar, Russia \\ ${ }^{2}$ Kuban State University, 149, Stavropol st., 350040, Krasnodar, Russia \\ ${ }^{3}$ Agrophysical Research Institute, 14, Grazhdanskiy pr., 195220, Saint-Petersburg, Russia
}

\begin{abstract}
The concept of digitalization of agricultural production in the Russian Federation provides for the implementation of measures to develop and create a system of geographic information monitoring and decision support in crop production. The aim of the research was to conduct geoinformation monitoring of rice crops to develop methods for automated mapping of their condition and yield forecasting. The studies were carried out on a test site of the Federal State Budgetary Scientific Institution "Federal Scientific Rice Centre" with an area of 274 hectares. The survey was performed by a quadcopter with a MicaSense RedEdge-M multispectral camera mounted on a fixed suspension. The shooting period using an unmanned aerial vehicle (UAV) was limited to early June and additionally used the Sentinel-2A satellite. To assess the state of rice crops, the normalized relative vegetative index NDVI was used. Based on the NDVI distribution and yield information from the combine TUCANO 580 (CLAAS), a statistical analysis was carried out in fields 7 and 9. Testing of the experimental methodology for monitoring crops in 2019 on the basis of remote sensing of test plots and geoinformation modeling and the statistical apparatus should be considered satisfactory.
\end{abstract}

\section{Introduction}

Krasnodar region is the main supplier of rice in Russia. In 2019, the volume of rice harvested in the Kuban amounted to more than 950 thousand tons, i.e. about $80 \%$ of the rice yield in our country. Hence, the significance of this crop for the agriculture of Kuban, including from the standpoint of food security of the country, is clear. With the development of modern remote sensing technologies, including the use of unmanned aerial vehicles (UAVs), the possibilities of assessing the condition of agricultural crops over large areas have significantly increased while reducing the cost of monitoring rice crops [1, 2]. The implementation of such technologies, coupled with appropriate techniques and the accumulation of arrays of experimental observations, can improve the quality of forecasting the yield of rice crops [6] and reduce the cost of land reclamation measures.

\footnotetext{
*Correspondent author: sma_49@mail.ru
} 
A large number of publications are devoted to the analysis of the state of large agricultural territories using satellite imagery of medium spatial resolution $[4,7,9-11,13]$. For monitoring rice crops, the normalized differential vegetative index NDVI and its varieties have been used for a long time. So, work [5] is devoted to finding statistical patterns of spectral brightness of rice crops, measured by ground methods, as well as vegetative and leaf indices obtained from an unmanned vehicle. A number of publications by Chinese researchers $[8,15]$ have implemented models for calculating rice yields based on the cumulative NDVI index based on data from UAV surveys for the entire growing season of the crop.

In this work, we analyze rice crops in the fields of the experimental production site of FSBSI "FSC of Rice" according to experimental surveys using an unmanned vehicle equipped with a multispectral camera. The obtained results lay the empirical basis for the subsequent development of a technique for automated mapping of the state of rice crops and forecasting rice yields in the conditions of Krasnodar region.

Aim of the study - conducting geoinformation monitoring of rice crops to develop methods for automated mapping of their condition and yield forecasting.

\section{Materials and Methods}

Rice fields on the territory of Krasnodar region, acting as test sites, are shown in Figure 1. We examined the test site of the Federal Scientific Rice Centre of 274 ha. The main cultivated crop is rice variety Favorit. The potential yield of the variety reaches 9-10 $\mathrm{t} / \mathrm{ha}$, the duration of the growing season is 112-117 days. The rice variety Favorit has a plant height of 95-105 cm.

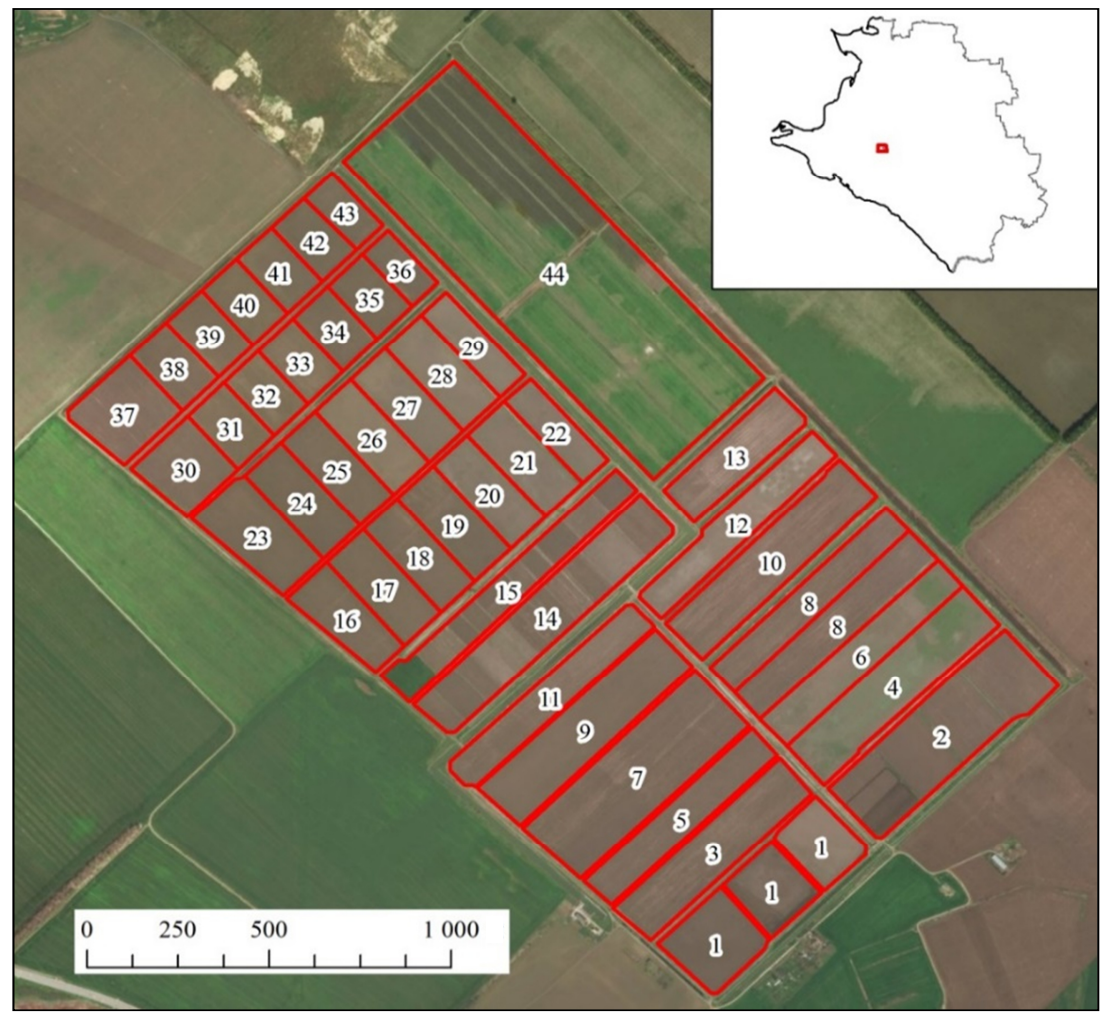

Fig. 1. Test site of Federal Scientific Rice Centre Substrate - Sentinel-2A Snapshot. 
The survey was performed by a quadrocopter with a MicaSense RedEdge-M multispectral camera (USA) mounted on a fixed suspension (Fig. 2). An autonomous flight was compiled taking into account the technical characteristics of the UAV and was carried out at an altitude of about $300 \mathrm{~m}$. Such a relatively high altitude affected the final performance with an insufficiently high spatial resolution. However, the choice of shooting height is dictated by the duration of the flight for a given area of the studied areas. Shooting dates: 05.28.2019, 06.06.2019, and 06.16.2019.

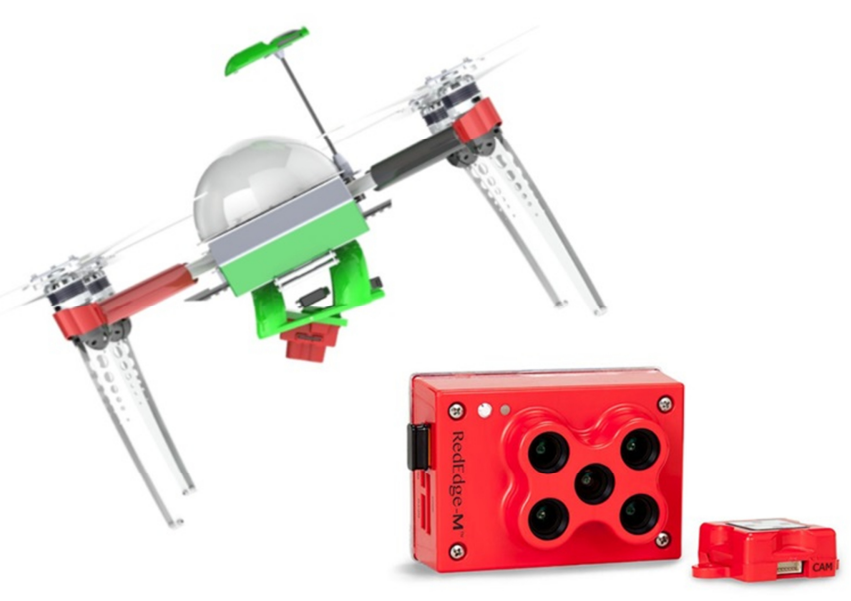

Fig. 2. Quadrocopter with camera MicaSense RedEdge-M.

The MicaSense RedEdge-M is a multispectral sensor; shooting materials are intended for constructing composite images, as well as pseudocolor combinations with the Red Edge channel, which allows achieving color separation of agricultural plants and weeds. Simultaneous shooting in five spectral ranges makes it possible to calculate a number of vegetation indices (NDVI, NDRE, etc.), and the light weight of the sensor (180 g) ensures compatibility with drones with a small payload. The main characteristics of the camera are shown in table 1 [12].

Table 1. The main technical characteristics of MicaSense RedEdge-M.

\begin{tabular}{|l|c|}
\hline \multicolumn{1}{|c|}{ Characteristic } & \multicolumn{1}{c|}{ Camera parameters } \\
\hline Weight & \multicolumn{1}{c|}{$231.9 \mathrm{~g}$} \\
\hline Field of View & $47.2^{\circ} \mathrm{HFOV}$ \\
\hline Spectral Bands & Blue, green, red, red edge, near-IR \\
\hline Ground Sample Distance & $8 \mathrm{~cm}$ per pixel (per band) at $120 \mathrm{~m}$ \\
\hline Capture Rate & 1 capture per second (all bands), 12-bit RAW \\
\hline
\end{tabular}

The data on the yield of the plots used in the analysis are vector layers for each of the fields of the test site in the form of a matrix with cells of $7 \times 7 \mathrm{~m}$. Each cell contains attributive data on the harvested rice yield according to the data of the TUCANO 580 combine (CLAAS) [3]. The article selectively analyzes the data of fields 1, 7, 9 (Fig. 1), which we adopted representative from the standpoint of productivity and values of indicators of the vegetation index.

Since the UAV survey period was limited for technical reasons by the beginning of June, we additionally used the Sentinel-2A satellite data covering the entire analyzed period (June 5, 2019 - August 29, 2019), which is useful for assessing the state of rice crops. The 
Sentinel family of satellites of the European Space Agency is designed to monitor the use of land, vegetation, forest and water resources. The spatial resolution of the images $(10 \mathrm{~m})$ involves the use of satellite data to assess the condition of crops within the boundaries of the studied fields. In addition, Sentinel-2A images were used as additional independent data to complement measurements from UAVs.

The materials taken by the MicaSense RedEdge-M sensor were processed in the Agisoft Photoscan software package (Russia). The processing included the following steps: aligning the images relative to each other, creating a dense cloud of points on their basis, building a digital terrain model and building an orthomosaic. The main end product is orthophotomaps with a spatial resolution of $20 \mathrm{~cm}$.

To assess the state of rice crops, the normalized relative vegetative index NDVI was used. The NDVI index was calculated in ArcGIS Desktop using the formula:

$$
N D V I=\frac{(N I R-R E D)}{(N I R+R E D)}
$$

where NIR is shooting in the near infrared spectrum (channel 5 of the RedEdge-M sensor), RED is shooting in the red zone of the spectrum (channel 3 of the RedEdge-M sensor).

\section{Results and Discussion}

For the correct performance of monitoring, operating with spatial data of successive temporal states of the object, a geodetic reference of the survey materials with an allowable error is required. The GPS receiver mounted on the UAV, when receiving geographical coordinates during the flight, provides an accuracy of 5-10 m. This requires additional reference. The latter was carried out manually for each data set according to the vector contours of the fields of the test polygon, taken as a standard. In the future, while continuing the experiment with the use of UAVs, it is necessary to survey the landfill with standard geodetic equipment.

The correct range of NDVI values is in the range of -1 to +1 . Vegetatively significant vegetation has NDVI values> 0.2; negative and initial positive values (up to 0.2) are represented by the surfaces of water bodies, building elements, open soil [14]. NDVI values $<0$ were not accepted by us for calculations. During processing, satellite images of Sentinel-2A are brought to the NDVI scale, comparable to images from UAVs.

After studying the distribution of NDVI and yield information, statistical analysis was limited to data from field 7 (Fig. 3). Vector yield data was converted to raster format in accordance with the similar parameters of NDVI data from both carriers (Fig. 4). 

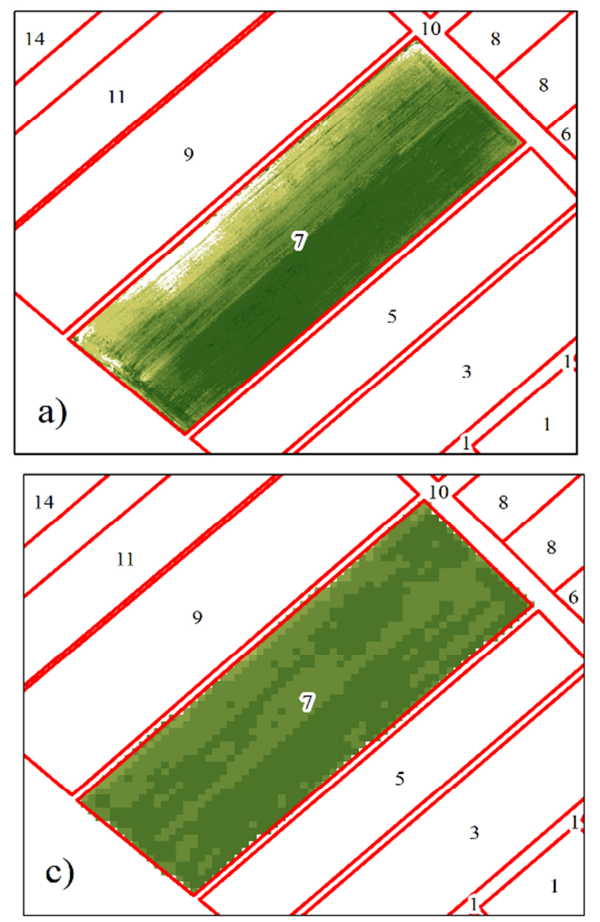

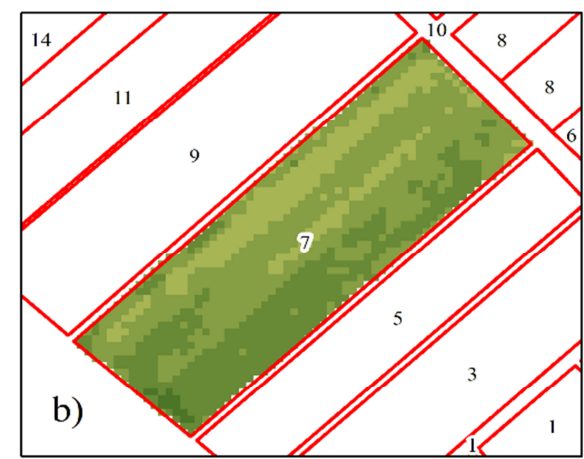

NDVI
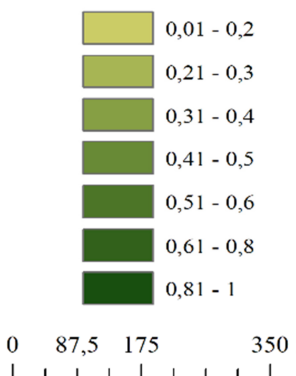

Fig. 3. Distribution of the normalized relative vegetative index NDVI in field 7 according to: a) UAVs (06.06.2019); b) satellite Sentinel-2A (06.05.2019); c) satellite Sentinel-2A (08.29.2019).

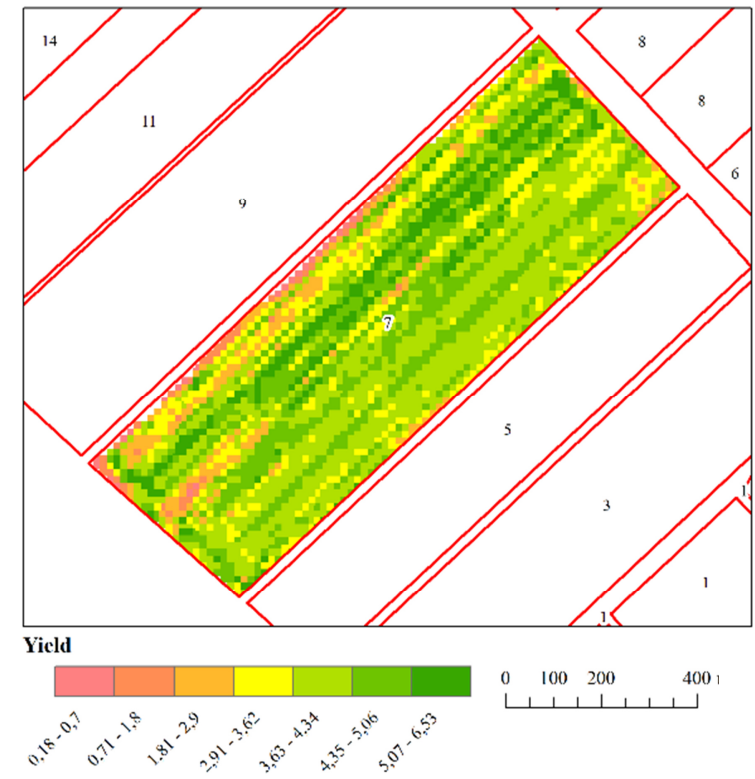

Fig. 4. Distribution of crop yields in field 7 ( $\mathrm{t} / \mathrm{ha})$.

Statistical data processing is performed separately for each field. Statistical analysis in the Statistica 6.0 program was preceded by the creation of a single table (Table 2) with the extracted numerical values of NDVI and yield from raster images. The table rows contain 
the coordinate description, attributive information on NDVI values for three periods according to the UAV and Sentinel satellite data, as well as yield values in each pixel.

Table 2. Source data extracted from raster images of analyzed fields (fragment).

\begin{tabular}{|c|c|c|c|c|c|}
\hline Coordinates X* & Coordinates Y & U06_NDVI7 & S06_NDVI7 & S08_NDVI7 & Yield7 \\
\hline 477767.357 & 4993072.775 & 0.45 & 0.31 & 0.44 & 4.7 \\
\hline 477776.748 & 4993072.775 & 0.42 & 0.44 & 0.43 & 5.04 \\
\hline 477786.139 & 4993072.775 & 0.43 & 0.38 & 0.50 & 4.98 \\
\hline 477795.53 & 4993072.775 & 0.46 & 0.31 & 0.52 & 5.15 \\
\hline 477804.921 & 4993072.775 & 0.47 & 0.30 & 0.51 & 4.65 \\
\hline 477814.312 & 4993072.775 & 0.48 & 0.32 & 0.50 & 4.76 \\
\hline 477823.702 & 4993072.775 & 0.47 & 0.33 & 0.52 & 4.82 \\
\hline 477833.093 & 4993072.775 & 0.46 & 0.32 & 0.51 & 4.54 \\
\hline 477842.484 & 4993072.775 & 0.44 & 0.32 & 0.51 & 4.98 \\
\hline 477851.875 & 4993072.775 & 0.35 & 0.31 & 0.53 & 5.04 \\
\hline 477861.266 & 4993072.775 & 0.31 & 0.31 & 0.52 & 4.82 \\
\hline 477870.657 & 4993072.775 & 0.25 & 0.30 & 0.51 & 5.15 \\
\hline 477880.048 & 4993072.775 & 0.27 & 0.31 & 0.50 & 5.21 \\
\hline 477889.439 & 4993072.775 & 0.27 & 0.31 & 0.50 & 5.15 \\
\hline 477898.83 & 4993072.775 & 0.25 & 0.31 & 0.51 & 4.26 \\
\hline 477908.221 & 4993072.775 & 0.25 & 0.31 & 0.54 & 4.37 \\
\hline 477917.612 & 4993072.775 & 0.19 & 0.32 & 0.54 & 4.59 \\
\hline 477927.003 & 4993072.775 & 0.15 & 0.31 & 0.56 & 4.14 \\
\hline 477936.394 & 4993072.775 & 0.16 & 0.32 & 0.54 & 4.70 \\
\hline 477945.785 & 4993072.775 & 0.22 & 0.34 & 0.48 & 4.48 \\
\hline
\end{tabular}

*Here: X, Y - coordinates of measurements in the Gauss-Kruger projection; U06NDVI7 - NDVI according to the UAV data (06.06.2019); S06-NDVI7 - NDVI according to Sentinel-2A (06/05/2019); S08-NDVI7 - NDVI according to Sentinel-2A (08/29/2019); Yield7 - productivity ( $\mathrm{t} / \mathrm{ha})$ (12-14.09.2019).

The statistical properties of the spatial distributions of the vegetation index and yield in the studied fields should be judged by the histograms (Fig. 5). From a brief overview of the type of frequency distribution of variables, the following preliminary conclusions can be made. In field 7 (area $13.75 \mathrm{ha}$ ), histograms reflecting the distribution of the vegetation index according to the survey data from UAVs and the Sentinel-2A satellite at close dates at the beginning of June have incomparable forms; unites their multimodality. The latter property, found on independent data, may indicate the presence of some specific microscale factors that determine the spatial distribution of rice crops inside the field in June. At the end of August, the character of the NDVI distribution changes, which is also confirmed by the measurement data on field 9 (Fig. 6): the histogram becomes close to unimodal, with some negative asymmetry inherent in both fields (the left side is elongated), especially noticeable according to field 7 Our expectations of the closeness of the histogram forms 
reflecting the distribution of NDVI and yield were more likely to be confirmed than were disproved. In both cases, on the analyzed fields, the forms are visually close. (Fig. 5, 6).
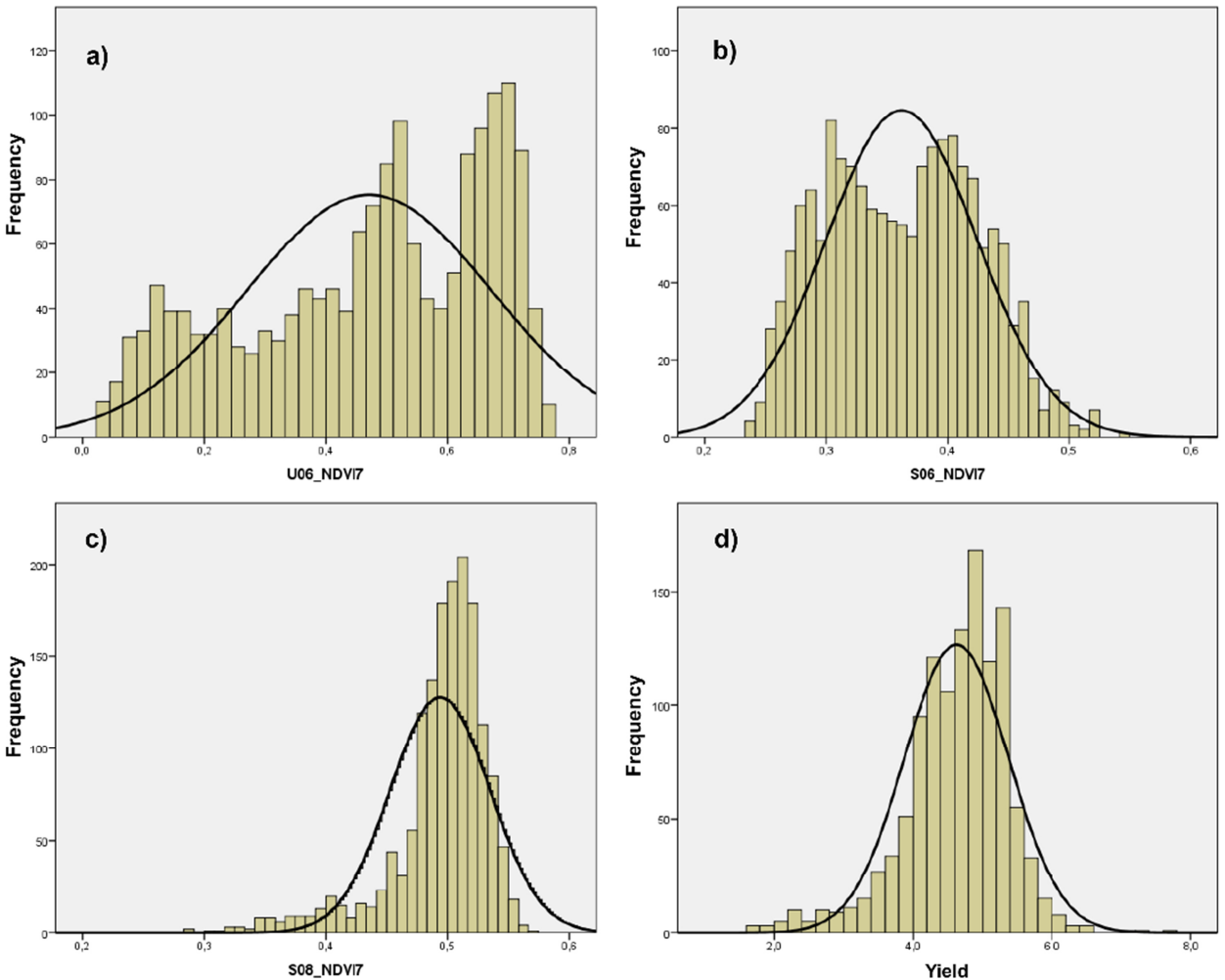

Fig. 5. Histograms of the distribution of the NDVI vegetation index on field 7 according to: a) BVS (06.06.2019), b) Sentinel-2A satellite (06.05.2019), c) Sentinel-2A satellite (08.29.2019), d) yield (September 12-14, 2019).

At the same time, analysis of the correlation matrix of NDVI variables showed a statistically moderate and weak connectivity of the spatial distributions of the studied variables (Table 3).

Studies based on the use of UAVs and Sentinel-2A satellite data make it possible to determine the degree of nitrogen supply to rice crops by optical indicators of their physiological state (NDVI), which are closely related to the yield map. Unmanned aerial vehicle (UAV) is a promising platform for remote sensing due to its versatility, and it is widely used to monitor the physiological state of rice crops and productivity [16]. Our data on the relationship of the optical-biological properties of rice crops with their productivity are generally consistent with reports of other authors $[17,18]$.

Table 3. The correlation matrix of NDVI values and yield indicators according to data from field 7.

\begin{tabular}{|l|c|c|c|c|}
\hline \multicolumn{1}{|c|}{ Variables* } & U06-NDVI7 & S06-NDVI7 & S08-NDVI7 & Yield7 \\
\hline U06-NDVI7 & 1 & 0.49 & 0.51 & 0.28 \\
\hline S06-NDVI7 & 0.49 & 1 & 0.24 & -0.05 \\
\hline S08-NDVI7 & 0.51 & 0.24 & 1 & 0.37 \\
\hline Yield7 & 0.28 & -0.05 & 0.37 & 1 \\
\hline
\end{tabular}


* Here: U06-NDVI7 - NDVI according to UAV data (06.06.2019); S06-NDVI7 - NDVI according to Sentinel-2A (06.05.2019); S08-NDVI7 - NDVI according to Sentinel-2A (29.08.2019); Yield7 - productivity (12-14.09.2019)

Thus, the possibility of determining the physiological state of rice crops by the optical properties of agrophytocenosis in the precision farming system is shown.

\section{Conclusions}

The results of the first measurements of the state of rice crops using unmanned aerial vehicles in Krasnodar region showed the promise of further experiment, while identifying the key technical features of its conduct and the need to comply with the correct requirements when shooting. The testing of the experimental methodology for monitoring crops in 2019, based on remote sensing means for test plots, a set of initial spatial data, geoinformation modeling tools and a statistical apparatus, should, in our opinion, be considered satisfactory. The monitoring experience gained allows us to hope for further development of the methodology up to its effectiveness in terms of operational and objective assessment of the state of rice crops in the Krasnodar Territory.

\section{Acknowledgment}

This work was financially supported by the RFBR grant 19-416-230021.

\section{References}

1. V. Badenko, D. Kurtener, V. Yakushev, A. Torbert, G. Badenko, Computational Science and Its Applications - ICCSA (2016) 10.1007/978-3-319-42111-76

2. V.P. Yakushev, E.V. Kanash, D.V. Rusakov, S.Yu. Blokhina, Advances in animal biosciences 8 (2017) Doi: 10.1017/ S204047001700053X

3. CLAAS Telematics, https://www.claas-telematics.com

4. Bo Duan, Shenghui Fang, Renshan Zhu, Frontiers in Plant Science 10 (2019) https://www.frontiers in.org/articles/10.3389/fpls.2019.00204/full

5. N. Hashimoto, Y. Saito, M. Maki, Remote Sensing Journal 11(11) (2019) https://www.mdpi.com/2072-4292/11/18/2119/htm

6. J. Huang, X. Wang, X. Li, PlOs One Journal 8 https://www.ncbi.nlm.nih.gov/pmc/articles /PMC3742684/

7. M. González-Betancourt, Z.L. Mayorga-Ruíz, DYNA 85, 47-56 (2018) https://www.researchgate.net/publication/325406776

8. X. Guan, Ch. Huang, G. Liu, Remote Sensing Journal 11(18) (2019) https://www.mdpi.com/2072-4292/11/18/2119/pdf

9. K. Lasko, K. Vadrevu Prasad, Tran V. Tuan, IEEE Journal of Selected Topics in Applied Earth Observations and Remote Sensing 11(2) (2018) https://ieeexplore.ieee.org/document/8253481

10. Li Songyang, Ding Xingzhong, Kuang Qianliang, Frontiers in Plant Science 9 (2018) https://www.frontiersin.org/articles/10.3389/ fpls.2018.01834/full

11. A. Yusup Liyantono, Y. Adillah, M. Yusuf Muhammad, IOP Conference Series: $\begin{array}{lllll}\text { Materials Science and } & 557 & \text { (2019) }\end{array}$ https://iopscience.iop.org/article/10.1088/1757-899X/557/1/012085/pdf 
12. MicaSense RedEdge-M User Manual, https://support.micasense.com/hc/enus/article_attachments/115004168274/RedEdge-M_User_Manual.pdf

13. F. Wang Fumin, W. Yao Zhang, Frontiers in Plant Science 10 (2019) https://www.ncbi.nlm.nih.gov/pmc/articles/PMC6468049

14. J. Weier, D. Herring, Measuring Vegetation (NDVI \& EVI) https://earthobservatory.nasa.gov/features/

15. X. Zhou, H.B. Zheng, X.Q. Xu, ISPRS Journal of Photogrammetry and Remote Sensing 130, 246-255 (2017) https://www.sciencedirect.com/science/article/abs/pii/ S0924271616302337

16. P.P. Rizky Aidil, L. Mohamad Solahudin, Procedia Environmental Sciences 24, 258265 (2015)

17. S. Huang, Y. Miao, F. Yuan, X. Ma, C. Tan et. al., Remote Sens. 7, 10646-10667 (2015) Doi:10.3390/RS70810646.

18. J. Lu, Y. Miao, W. Shi, J. Li., F. Yuan, Sci. Rep. 7, 14073 (2017) 